University of Nebraska - Lincoln

DigitalCommons@University of Nebraska - Lincoln

John R. Hardy Papers

Research Papers in Physics and Astronomy

$8-1-2000$

\title{
Molecular-Dynamics Study of Phase Transitions in Alkali Thiocyanates
}

\author{
M. M. Ossowski \\ University of Nebraska-Lincoln \\ John R. Hardy \\ University of Nebraska-Lincoln \\ R. W. Smith \\ University of Nebraska at Omaha, robertsmith@unomaha.edu
}

Follow this and additional works at: https://digitalcommons.unl.edu/physicshardy

Part of the Physics Commons

Ossowski, M. M.; Hardy, John R.; and Smith, R. W., "Molecular-Dynamics Study of Phase Transitions in Alkali Thiocyanates" (2000). John R. Hardy Papers. 69.

https://digitalcommons.unl.edu/physicshardy/69

This Article is brought to you for free and open access by the Research Papers in Physics and Astronomy at DigitalCommons@University of Nebraska - Lincoln. It has been accepted for inclusion in John R. Hardy Papers by an authorized administrator of DigitalCommons@University of Nebraska - Lincoln. 


\title{
Molecular-dynamics study of phase transitions in alkali thiocyanates
}

\author{
M. M. Ossowski and J. R. Hardy \\ Department of Physics and Center for Electro-Optics, University of Nebraska, Lincoln, Nebraska 68588-0111 \\ R. W. Smith \\ Department of Chemistry, University of Nebraska, Omaha, Nebraska 68182-0109
}

(Received 22 November 1999)

\begin{abstract}
An account is presented of our studies of the order-disorder phase transitions in KSCN, RbSCN, and CsSCN. These are based on parameter-free interionic potentials based on the Gordon-Kim modified electron gas formalism extended to molecular ions. We performed static structural relaxations and supercell molecular dynamics and predicted with reasonable accuracy the temperatures for the onset of the transitions. In particular, we address the question of how the $\mathrm{SCN}^{-}$ions disorder to yield subsequent structural transformations. We found high-temperature phases of average $F m \overline{3} m$ symmetry for both KSCN and RbSCN. We argue that in reality the full appearance of these phases is preempted by melting. However, they are candidates for the twin boundaries which are observed in the high-temperature "average" tetragonal phases. The high-temperature phase of CsSCN was found to be of average $F m \overline{3} m$ symmetry.
\end{abstract}

\section{INTRODUCTION}

We have recently presented results of our moleculardynamics (MD) studies of selected alkali azides ${ }^{1}$ (ionic solids with linear $\mathrm{N}_{3}{ }^{-}$anions), and it is now appropriate to extend such work to near-isomorphous $\mathrm{SCN}^{-}$compounds. Alkali thiocyanates, however, due to the mass and charge asymmetry of $\mathrm{SCN}^{-}$ions, have a different dynamics, as "free" rotations of $\mathrm{SCN}^{-}$groups about their centers of mass (c.m.) are not permitted. Also, a large $S$ center tends to "anchor" the $\mathrm{SCN}^{-}$group: i.e., $\mathrm{SCN}^{-}$tends to want to rotate about $S$. Unlike in azides, this intrinsically more complex dynamics does not allow us to decouple the c.m. translational motion of the $\mathrm{SCN}^{-}$ion from its rotation about the c.m. It is our goal to give a microscopic view of how such dynamics of $\mathrm{SCN}^{-}$motion drives phase transitions in alkali thiocyanates.

\section{EXPERIMENTAL PHASE DIAGRAM OF KSCN, RbSCN, and CsSCN}

This work concentrates on three selected thiocyantes: $\mathrm{KSCN}, \mathrm{RbSCN}$, and CsSCN. Experimentally, the lowtemperature phase of KSCN has orthorhombic $\mathrm{Pbcm}$ symmetry (related to the $\mathrm{CsCl}$ structure) with $Z=4 .^{2}$ In this phase thiocyanate groups are perfectly ordered along two distinctive crystallographic directions. Although the lowtemperature phase of $\mathrm{RbSCN}$ is believed to be isomorphous with that of KSCN, no detailed analysis of the crystal structure of this phase has yet been carried out. ${ }^{3}$ As the temperature is raised, at ambient pressure, $\mathrm{KSCN}$ and $\mathrm{RbSCN}$ transform at 413 and $435 \mathrm{~K}$, respectively, into rotationally disordered phases characterized by random orientation of the $\mathrm{SCN}^{-}$ions with respect to the edges of the high-temperature unit cells. This high-temperature phase of $\mathrm{KSCN}$ and $\mathrm{RbSCN}$ is believed to have the average tetragonal $I 4 / \mathrm{mcm}$ symmetry (also related to the $\mathrm{CsCl}$ structure) with $Z=2$. There remains, however, disagreement whether during the transition $\mathrm{SCN}^{-}$ions move in or out of the $\mathrm{SCN}^{-}$plane. ${ }^{4,5} \mathrm{In}$ addition, it was recently suggested that the high-temperature phase of KSCN and RbSCN may not be a true rotationally disordered phase, but instead is an ensemble of ordered orthorhombic microdomains with a local ordered configuration of $\mathrm{SCN}^{-}$ions. ${ }^{6,7}$

The low-temperature phase of CsSCN has an orthorhombic Pnma symmetry (related to the $\mathrm{NaCl}$ structure) with $Z$ $=4 .{ }^{8}$ In contrast to the low-temperature phases of KSCN and $\mathrm{RbSCN}$, the larger $\mathrm{Cs}^{+}$cations are now pressed into the $\mathrm{SCN}^{-}$layers. As the temperature is raised, at ambient pressure, CsSCN undergoes at $470 \mathrm{~K}$ a disordering phase transition characterized, as in $\mathrm{KSCN}$ and $\mathrm{RbSCN}$, by a random orientation of the $\mathrm{SCN}^{-}$ions with respect to the edges of the high-temperature unit cell. Experimental results point to the average $P m \overline{3} m$ (CsCl-like) symmetry with $Z=1{ }^{8}$

\section{STATIC RELAXATION OF THE LOW-TEMPERATURE STRUCTURE}

We follow the computational algorithm developed earlier and used successfully to study a broad variety of molecular ionic solids, including the azides. ${ }^{1}$ Our method is founded on parameter-free interionic potentials based on the Gordon-Kim ${ }^{9}$ (GK) modified electron gas formalism extended to molecular ions. Since this approach was repeatedly discussed in our previous work, ${ }^{1,10}$ it is appropriate here to only sketch the procedure as it relates to the thiocyanates.

To compute short-range GK pair potentials, we first performed quantum-chemistry geometry optimization for the whole $\mathrm{SCN}^{-}$ion using Hartree-Fock algorithm with the standard $6-31 \mathrm{G}^{*}$ basis for all three atoms as supported by the GAUSSIAN 94 (Ref. 11) commercial package. We then decomposed the resulting charge density for the optimized $\mathrm{SCN}^{-}$ ion into the $\mathrm{S}, \mathrm{C}$, and $\mathrm{N}$ centers in the spirit of a Mulliken population analysis. ${ }^{12}$ The intraionic potentials were treated within the harmonic approximation whose coefficients were determined by the GAUSSIAN 94 program, while the longrange potentials were computed from the fractional ionicities of +0.1023 on $\mathrm{C},-0.5526$ on $\mathrm{S},-0.5497$ on $\mathrm{N}$, and +1 on alkali cations. 
TABLE I. Prototypic atomic positions in the relaxed structures of KSCN, RbSCN and CsSCN. Experimental values are given in parentheses (Refs. 15 and 16).

\begin{tabular}{ccccc}
\hline \hline Prototype & $x / a$ & $y / b$ & $z / c$ & Wyckoff \\
\hline K in KSCN & $0.2142(0.2059)$ & 0.25 & 0 & $4(c)$ \\
S in KSCN & $0.6066(0.6033)$ & $0.3099(0.2919)$ & 0.25 & $4(d)$ \\
C in KSCN & $0.7938(0.7737)$ & $0.3099(0.2919)$ & 0.25 & $4(d)$ \\
N in KSCN & $0.9227(0.8934)$ & $0.4365(0.4139)$ & 0.25 & $4(d)$ \\
Rb in RbSCN & 0.2166 & 0.25 & 0 & $4(c)$ \\
S in RbSCN & 0.6104 & 0.1303 & 0.25 & $4(d)$ \\
C in RbSCN & 0.7941 & 0.3108 & 0.25 & $4(d)$ \\
N in RbSCN & 0.9204 & 0.4340 & 0.25 & $4(d)$ \\
Cs in CsSCN & $0.1907(0.1781)$ & 0.25 & $0.0863(0.1060)$ & $4(c)$ \\
S in CsSCN & $0.0267(0.0187)$ & 0.25 & $0.6878(0.6899)$ & $4(c)$ \\
C in CsSCN & $0.1788(0.1724)$ & 0.25 & $0.5369(0.5588)$ & $4(c)$ \\
N in CsSCN & $0.2864(0.2849)$ & 0.25 & $0.4366(0.4681)$ & $4(c)$ \\
\hline \hline
\end{tabular}

With such developed potential-energy surfaces, we performed static relaxation for the room-temperature phases of $\mathrm{KSCN}, \mathrm{RbSCN}$, and $\mathrm{CsSCN}$. We used periodic boundary conditions for a Newton-Raphson-type minimization and standard Ewald summation of the Coulomb forces.

In all three cases $\mathrm{KSCN}, \mathrm{RbSCN}$, and $\mathrm{CsSCN}$, we used cells equivalent to the crystallographic unit cells for the room-temperature phases of these systems. For RbSCN, however, we could not find a full set of experimental structural data, and thus we used fractional atomic positions for KSCN combined with known lattice parameters for RbSCN and regarded these two structures as isomorphous. ${ }^{3}$ Table I lists prototypic atomic positions and lattice constants obtained in static relaxations with $\mathrm{Pbcm}$ (KSCN and RbSCN) and Pnma (CsSCN) symmetry constraints and compares them with experiment. For all three thiocyanates the agreement in lattice constants is very good. An average $\sim 5 \%$ shortening of the lattice constants in our relaxations has been a common feature in our work with GK potentials. ${ }^{10}$ It must also be noted that the structural data in Table I are for room temperature and thus include the effects of thermal expansion. The largest differences in fractional parameters in KSCN and CsSCN correspond to distortions well within the thermal fluctuations of atoms at room temperature. We are therefore confident that our relaxation procedure and potentials are reproducing the "true" low-temperature phase of $\mathrm{RbSCN}$.

We also performed static relaxation for the three thiocyanates without symmetry constraints of the low-temperature phases. In all cases the resultant structures were exactly those given by relaxation with the constraints.

\section{MOLECULAR-DYNAMICS SIMULATIONS OF THE PHASE TRANSITIONS IN KSCN AND RbSCN}

Good agreement of the statically relaxed low-temperature structures of $\mathrm{KSCN}, \mathrm{RbSCN}$, and $\mathrm{CsSCN}$ with experiment served as a test for the validity of our GK pair potentials. We subsequently employed these potentials in our MD simulations which followed a constant-energy modified Verlet algorithm with periodic boundary conditions and Ewald summation for lattice energy and forces. We used supercells of 128 atoms obtained from doubling the statically relaxed unit cells in all three directions.
As the first step, we removed any residual energy by "quenching"' the supercells to $0 \mathrm{~K}$. We then quickly heated them to temperatures $\sim 50 \mathrm{~K}$ below experimental $T_{c}$ 's and finally let them equilibrate for $25 \mathrm{ps}$. Consequently, we "'heated"' the supercells in steps equivalent to $\sim 5 \mathrm{~K}$ and let them equilibrate for $12 \mathrm{ps}$ after each pulse. A typical run with a MD time step of 0.001 ps from $0 \mathrm{~K}$ to $\sim 1000 \mathrm{~K}$ on one R10000 CPU running at $195 \mathrm{MHz}$ took about 1 week.

We first performed MD simulations on $\mathrm{KSCN}$ and RbSCN, which are isomorphous in their low-temperature phase. Figure 1 shows lattice constants as a function of temperature for a $\mathrm{MD}$ run on $\mathrm{KSCN}$ within a temperature range between $\sim 350 \mathrm{~K}$ and "melting", above $\sim 600 \mathrm{~K}$, while Fig. 2 shows in- $(a b)$ and out-of- ( $b c$ and $c a$ ) plane average cross sections for the ordered KSCN system as it approaches $T_{c}$. Large ovals centered about atoms represent "thermal ellipsoids" which indicate rms deviation of atoms from their average positions. We see that the system is ordered, of a layered structure, with a slight tendency of the $\mathrm{SCN}^{-}$groups to unlock out of the $a b$ plane. Just above $T_{c}$ the structure changes radically; specifically, the $\mathrm{SCN}^{-}$groups appear to rotate into the $a c$ and $b c$ planes and the $c$ axis begins to dilate rapidly. This behavior is depicted in Fig. 3, which shows large thermal ellipsoids associated with concurrent translational motion of $\mathrm{K}^{+}$and $\mathrm{CSN}^{-}$ions as the system undergoes

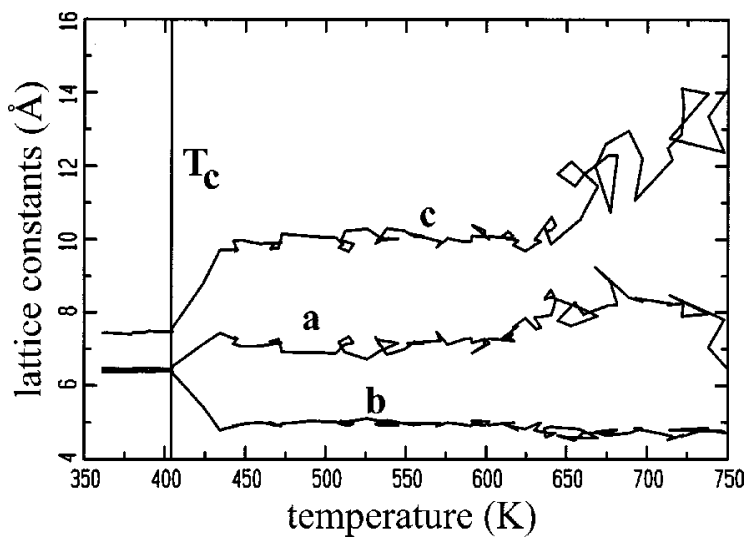

FIG. 1. Lattice constants as a function of temperature for the MD run on KSCN. See Figs. 2-4 and Ref. 1 for the description of the structural transformation. 

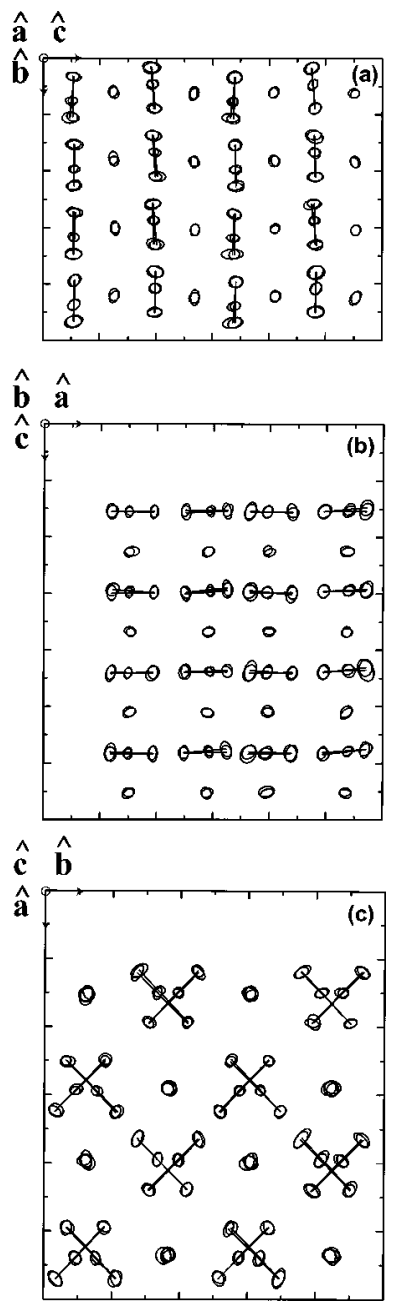

FIG. 2. Projections of the atomic positions along orthorhombic axes in the average structure of KSCN obtained from MD simulations at $404 \mathrm{~K}$. The "thermal ellipsoids", indicate the rms deviation of atoms from their average positions and represent thermal motions of the atoms. Bonds connect the average positions of the center carbon with end nitrogen and sulphur atoms in the $\mathrm{SCN}^{-}$ion.

a sc $\rightarrow$ fcc transformation very similar to that which we have reported for the near-isomorphous azides. ${ }^{1}$ In the process the volume-ion pair increases by $\sim 15 \%$, an amount much larger than one expects for melting. Hence we expect again, as for the azides, that melting will in reality preempt the crystal restructuring. However, experimentally there exists nominally tetragonal phase over $\sim 30 \mathrm{~K}$ below melting, which we apparently do not observe.

This paradox may be resolved by the further experimental finding that this "tetragonal" phase is not truly tetragonal at all; rather, it appears to consist of dynamically moving "domains" of orthorhombic material with antiphase boundaries between them so that the average symmetry appears tetragonal. ${ }^{6}$ Our model of disorder $\rightarrow$ immediate melting, however, implicitly assumes uniformity, which, by the very nature of periodic boundary conditions and the smallness of our supercell, is imposed automatically on our simulations. However, if we relax this assumption and consider the possibility of the domain state in which disorder is confined to the interfaces between domains, then it is entirely possible to have an intermediate solid state, prior to melting, in which,
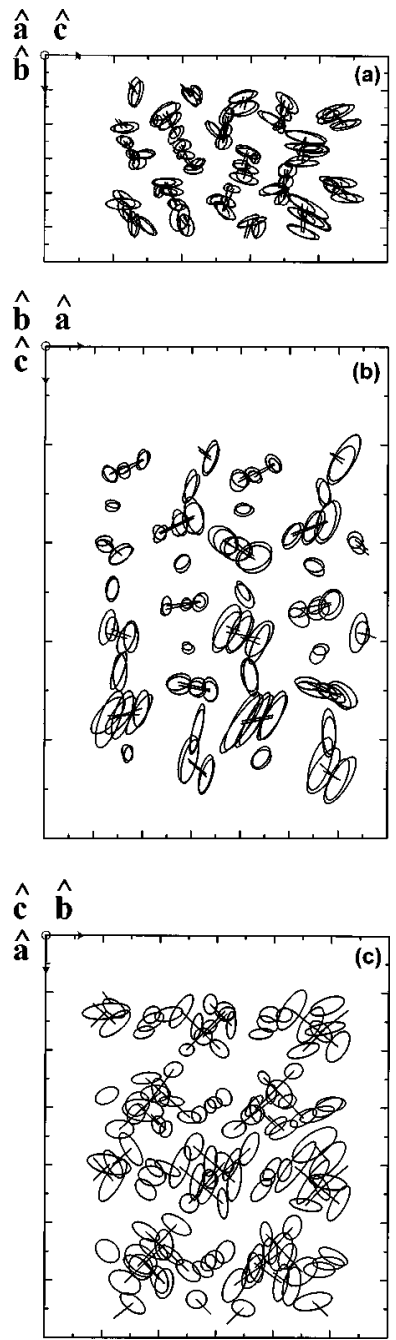

FIG. 3. Projections of the atomic positions along orthorhombic axes in the average structure of KSCN obtained from MD simulations at $424 \mathrm{~K}$. The elongated "thermal ellipsoids" indicate the onset of structural transformation from the sc-like to the fcc-like phase.

because only a small factor of the lattice is disordered, the expansion along $\mathbf{c}$ is substantially less and only when the domain boundary reaches some critical value does the crystal melt.

Indeed, a detailed examination of average cross sections at temperatures above $T_{c}$, like the ones in Fig. 4, reveals that over a time of $\sim 25 \mathrm{ps}$ some $\mathrm{SCN}^{-}$groups tend to reorient between $+c$ and $-c$. This, together with NMR (Ref. 13) findings that the extremely slow head-to-tail flipping of $\mathrm{SCN}^{-}$groups is a dynamic effect, suggests that our fluctuating structure may well be that existing within the antiphase domain boundaries and the "real" phase transition is triggered by its appearance which permits the observed antiphase boundaries to form.

In $\mathrm{RbSCN}$ our simulations led to similar findings as in KSCN, RbSCN also restructured to an average $F m \overline{3} m$ phase with a volume-ion pair expansion of $\sim 13 \%$ as a consequence of the unlocking of the rotational motion of $\mathrm{SCN}^{-}$ions. As in $\mathrm{KSCN}$, we also found that the $\mathrm{SCN}^{-}$fluctuations were slow and predominantly out of plane. The main difference between RbSCN and KSCN is that in RbSCN the restructur- 

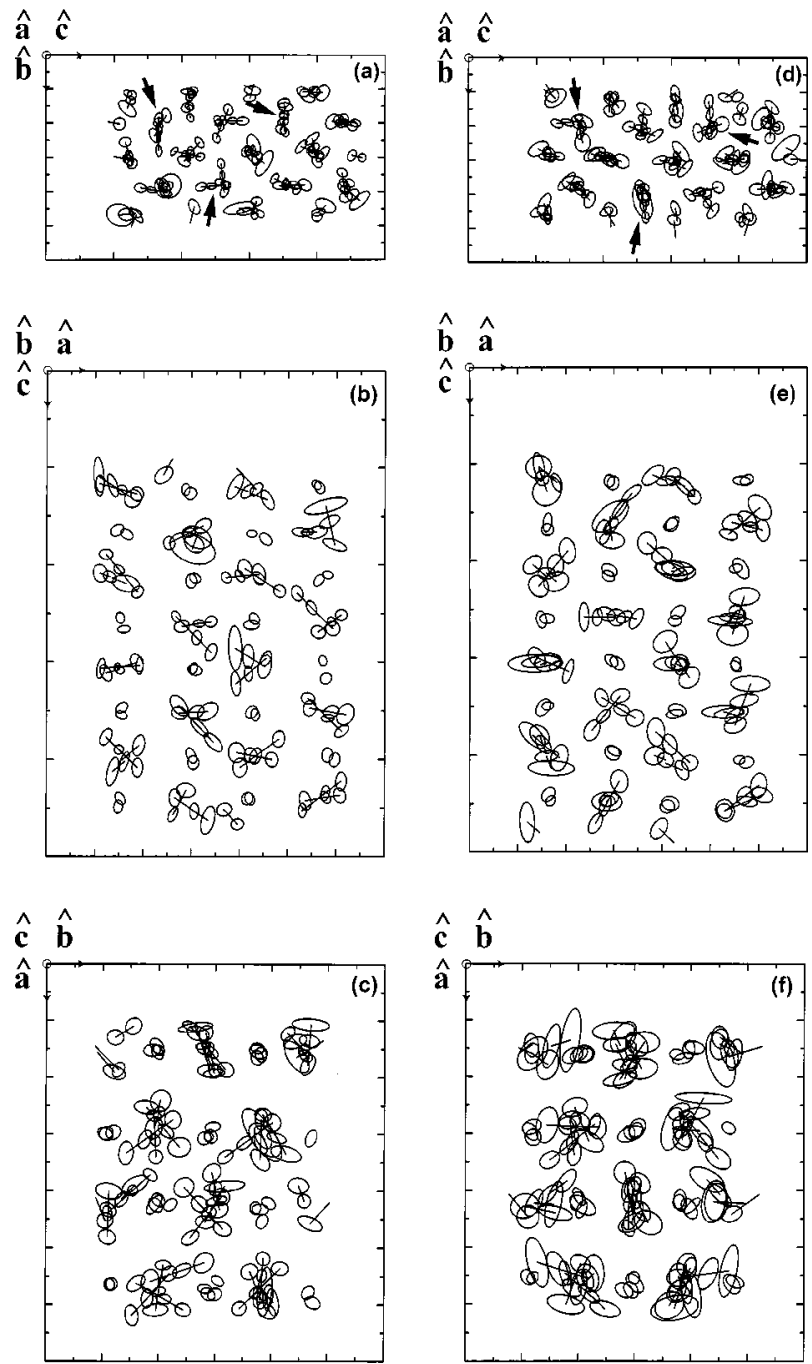

FIG. 4. Projections of the atomic positions along orthorhombic axes in the average structure of KSCN obtained from MD simulations at $443 \mathrm{~K}$ (a), (b), (c) and $448 \mathrm{~K}$ (d), (e), (f) and separated by $\sim 25 \mathrm{ps}$ in the MD run. Arrows in the projections along $a$ axis indicate examples of $\mathrm{SCN}^{-}$groups reorienting between $+c$ and $-c$.

ing to the average fcc phase was somewhat separated from the onset of rotational disordering at $T_{c}$, as illustrated in Fig. 5 , whereas in KSCN these two actions commenced almost simultaneously. This difference between the critical behavior of $\mathrm{K}^{+}$and $\mathrm{Rb}^{+}$systems was observed previously in our study of azides. ${ }^{1}$ Table II lists theoretical values of $T_{c}$ for $\mathrm{KSCN}, \mathrm{RbSCN}$, and $\mathrm{CsSCN}$ (discussed in the next section) and compares them with experimental data.

\section{MOLECULAR-DYNAMICS SIMULATIONS OF THE PHASE TRANSITION IN CsSCN}

$\mathrm{CsSCN}$ is different from $\mathrm{KSCN}$ and $\mathrm{RbSCN}$ in that its low-temperature phase is of $\mathrm{NaCl}$ and not $\mathrm{CsCl}$ type. Moreover, the domain structure both below and above $T_{c}$ in $\mathrm{CsSCN}$ is absent. ${ }^{14}$ We may therefore expect that the intrinsically stable (average fcc) high-temperature phase in $\mathrm{CsSCN}$ is reached without such major restructuring as in $\mathrm{KSCN}$ and $\mathrm{RbSCN}$ and that this high-temperature phase should have its experimental counterpart.
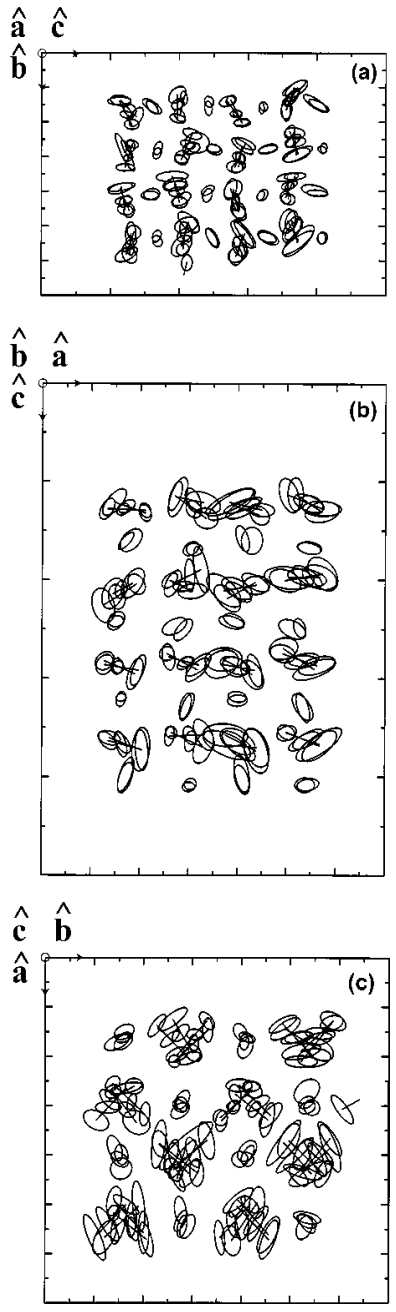

FIG. 5. Projections of the atomic positions along orthorhombic axes in the average structure of RbSCN obtained from MD simulations at $411 \mathrm{~K}$ after the temperature dropped from $433 \mathrm{~K}$ at $T_{c}$. As in the case of $\mathrm{KSCN}$, the elongated thermal ellipsoids indicate the onset of the structural transformation from the sc-like to the fcclike phase. However, unlike in $\mathrm{KSCN}$, the structure in these cross sections has not yet transformed. This reflects the fact that in $\mathrm{RbSCN}$ the restructuring to the average fcc phase is delayed, compared with that in KSCN.

Figure 6 shows lattice constants as a function of temperature for a $\mathrm{MD}$ run on $\mathrm{CsSCN}$ within a temperature range between $\sim 350 \mathrm{~K}$ and "melting", above $\sim 600 \mathrm{~K}$. Just below $T_{c}$ our simulation shows an unlocking of the rotational motion of the $\mathrm{SCN}^{-}$ions, which, as the temperature is raised, becomes uniform free rotations. ${ }^{8}$ Consequently, the supercell

TABLE II. Calculated and experimental values of $T_{c}$ for the restructuring transformations together with observed melting points for KSCN, RbSCN and CsSCN (Refs. 2, 3, 15, and 17). For RbSCN and CsSCN, the values of $T_{c}$ are at the upper bound limits of the hystereses of $\sim 20$ and $\sim 80 \mathrm{~K}$, respectively.

\begin{tabular}{cccr}
\hline \hline & KSCN & RbSCN & CsSCN \\
\hline$T_{c}$ theory $(\mathrm{K})$ & 405 & 433 & 647 \\
$T_{c}$ experiment $(\mathrm{K})$ & 413 & 435 & 470 \\
Melting experiment $(\mathrm{K})$ & 445 & 457 & $\geqslant 479$ \\
\hline \hline
\end{tabular}




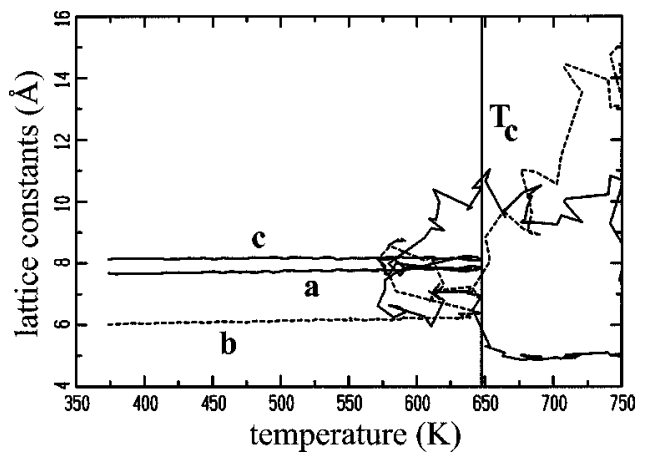

FIG. 6. Lattice constants as a function of temperature for the MD run on CsSCN. See text and Fig. 7 for a description of the structural transformation.

undergoes a structural transformation to an average $F m \overline{3} m$ high-temperature phase as illustrated in Fig. 7. In view of a report of CsSCN transforming to the average CsCl-like hightemperature phase, ${ }^{8}$ we did a simulation starting from the CsCl-like low-temperature structure of the other thiocyanates and found that this phase also transforms to the average $F m \overline{3} m$ phase in which $\mathrm{SCN}^{-}$ions are completely disordered. We also computed the static energy of CsSCN in the orthorhombic $\mathrm{Pbcm}$ phase and found that this new structure is $\sim 0.1 \mathrm{eV} / f . u$. higher in energy than the observed structure. This indicates that perhaps it would be profitable to perform a redetermination of the crystal structure of the hightemperature phase of CsSCN.

Unlike for $\mathrm{KSCN}$ and $\mathrm{RbSCN}$, the $T_{c}$ calculated for $\mathrm{CsSCN}$ is significantly higher than the experimental value. However, neutron diffraction studies of CsSCN (Ref. 15) suggest that there is a marked activation barrier to the transition, as there is no evidence of a transformation up to $T_{c}$. Hence our " $T_{c}$ " may represent the temperature at which activation is possible rather than equilibrium: i.e., we have in the case of CsSCN a substantial overheating.

\section{CONCLUSION}

We have employed our $a b$ initio potentials to reproduce the low-temperature phases of KSCN and CsSCN to a high degree of accuracy. In addition, we determined the lowtemperature structure of $\mathrm{RbSCN}$. We subsequently proceeded to a molecular-dynamics simulation of the orderdisorder phase transitions in these materials, which gave us a microscopic view of how the structural transformations are driven by the onset of hindered rotations of $\mathrm{SCN}^{-}$ions. In particular, we determined that KSCN and RbSCN undergo a major restructuring to average fcc phases above the $T_{c}$. As for $\mathrm{KN}_{3}$ and $\mathrm{RbN}_{3},{ }^{1}$ we argue that, in reality, melting preempts such structural transformations. It is entirely possible, however, as we have discussed, that these high-temperature rotationally disordered fcc phases are associated with observed antiphase orthorhombic domain boundaries above the $T_{c}$. CsSCN undergoes a minor restructuring to an average fcc phase and not to an average sc phase, as previously believed.

\section{ACKNOWLEDGMENT}

This work was supported by the U.S. Army Research Office under Grant Nos. DAAG 55-97-1-0106 and DAAG 5598-1-0273.

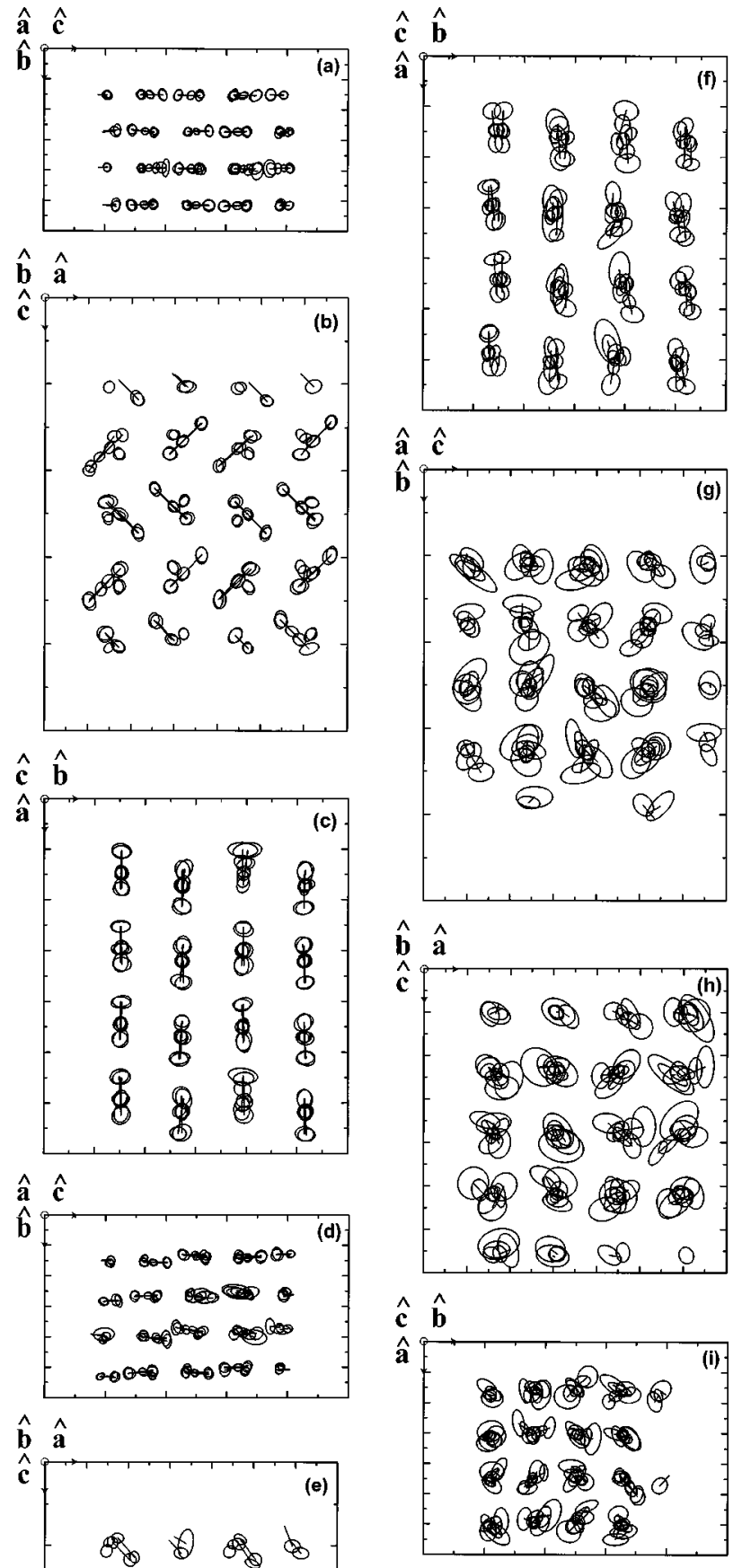

FIG. 7. Projections of the atomic positions along orthorhombic axes in the average structure of CsSCN obtained from MD simulations at $647 \mathrm{~K}$ before the sample reached $T_{c}$ (a), (b), (c), at $636 \mathrm{~K}$ after the sample reached $T_{c}(\mathrm{~d})$, (e), (f), and $577 \mathrm{~K}$ after the sample has relaxed in the new fcc-like phase $(\mathrm{g}),(\mathrm{h}),(\mathrm{i})$. 
${ }^{1}$ M. M. Ossowski, J. R. Hardy, and R. W. Smith, Phys. Rev. B 60, 15094 (1999).

${ }^{2}$ S. Yamamoto, M. Sakuno, and Y. Shinnaka, J. Phys. Soc. Jpn. 56, 2604 (1987).

${ }^{3}$ W. Klement, Jr. and C. W. F. T. Pistorius, Bull. Chem. Soc. Jpn. 49, 2148 (1976).

${ }^{4}$ W. Schranz, Phase Transit. 51, 1 (1994).

${ }^{5}$ A. Fuith, Phase Transit. 62, 1 (1997).

${ }^{6}$ O. Blashko, W. Schwarz, W. Schranz, and A. Fuith, Phys. Rev. B 44, 9159 (1991).

${ }^{7}$ O. Blashko, W. Schwarz, W. Schranz, and A. Fuith, J. Phys.: Condens. Matter 6, 3469 (1994).

${ }^{8}$ S. Manolatos, M. Tillinger, and B. Post, J. Solid State Chem. 7, $31(1973)$
${ }^{9}$ R. G. Gordon and Y. S. Kim, J. Chem. Phys. 56, 3122 (1971).

${ }^{10}$ H. M. Lu and J. R. Hardy, Phys. Rev. B 42, 8339 (1990).

${ }^{11}$ M. J. Frish et al., computer code GAUSSIAN 94, Gaussian, Inc., Pittsburgh, PA, 1994.

${ }^{12}$ R. S. Mulliken, J. Chem. Phys. 23, 1833 (1955).

${ }^{13}$ R. Blinc, T. Apih, A. Fricelj, J. Dolinsek, J. Seliger, A. Fuith, W. Schranz, H. Warhanek, and D. C. Ailion, Europhys. Lett. 39, 627 (1997).

${ }^{14}$ R. Blinc, T. Apih, A. Fuith, and H. Warhanek, Solid State Commun. 94, 983 (1995).

${ }^{15}$ B. K. Moss, S. L. Mair, G. J. McIntyre, and R. K. McMullan, Acta Crystallogr., Sect. B: Struct. Sci. 43, 16 (1987).

${ }^{16}$ C. Akers, S. W. Peterson, and R. D. Willett, Acta Crystallogr., Sect. B: Struct. Crystallogr. Cryst. Chem. 24, 1125 (1968).

${ }^{17}$ F. J. Owens, Solid State Commun. 29, 789 (1979). 